\title{
Estimation of Packet Loss on MAC Layer in IEEE 802.11 Wireless Local Area Networks
}

\author{
Shafi Shahsavar Mirza ${ }^{1}$ and Khaled M. Almustafa ${ }^{2}$ \\ ${ }^{1}$ Western University, Canada \\ ${ }^{2}$ Prince Sultan University. Riyadh, K.S.A \\ ${ }^{1}$ shafimirza2@gmail.com, ${ }^{2}$ kalmustafa@psu.edu.sa
}

\begin{abstract}
In wireless local area networks, management decisions concerning channel selection, rate selection, and power management have a profound impact on the throughput performance, particularly in dense areas. These measurements, however, are too weakly associated with the MAC layer to be able to make such management decisions. Therefore, direct measurements of the channel quality at the MAC layer are necessary to facilitate appropriate decision-making activities. Estimating quantities, such as the probability of collision and the probability of channel-induced errors, will enable the MAC layer to make suitable decisions1. This paper proposes a mechanism to calculate both the channel error probability and collision probability through the methodology of distribution. Some scenarios are discussed to demonstrate the effectiveness of the measurements.
\end{abstract}

Keyword: MAC Layer, IEEE 802.11, WLAN, Estimation

\section{Introduction}

Wireless Local Area Networks (WLANs) can be mobilized in an ad hoc manner without any need for infrastructure [1]. Because there has been growing interest in WLANs, IEEE has formed 802.11 study groups to recommend standards for the wireless medium [2]. This group is responsible for specifying standards for media access control (MAC) and physical (PHY) layers of the OSI (Open Systems Interconnection) reference model. The basic 802.11 MAC protocol is a Distributed Coordination Function (DCF) component that operates as a listen-before-talk scheme, based on the Carrier Sense Multiple Access (CSMA) [3-6].

According to the 802.11 protocol, the working group established an activity to enhance the current 802.11 MAC schematic to support applications with QoS requirements, and to provide security. Such a network could present a variety of opportunities for new multimedia applications on mobile/portable devices. WLAN Management decisionssuch as channel selection, rate selection, and power control-have profound impacts on the throughput performance, particularly in dense areas [7-9]. Management decisions are generally undertaken according to the observed Signal to Noise Ratio (SNR) at the physical layer. The measurements taken within the physical layer are weakly correlated to the MAC layer [10].

This means that a direct measurement of the channel quality at the MAC layer is necessary to make a relevant decision.

This paper presents two WLAN scenarios: one pertains to the infrastructure basic service set (BSS), and the other focuses on the independent basic service set (IBSS). The

1 Part of this work will be presented at the International Conference on Engineering and Information Technology (ICEIT 2015), Singapore, Jan. 19-22, 2015. 
main difference between these two scenarios is that in the infrastructure basic service set there will be an access point that manages all the flow, whereas in the independent basic service set there will be no access point to maintain the flow of traffic. The infrastructure basic service set is composed of an access point (AP) and a number of stations associated with this access point. The access point provides a connection between the stations and the infrastructure.

These wireless LAN access points ensure network contact is never lost. This access point transmits data between the WLAN and the wired network, and each single wireless access point can support simultaneous users. In WLAN networks, the cost depends on the number of wireless LAN access points deployed. In other words, the number of wireless LAN access points is proportionate to the required coverage within the region. The number of wireless LAN access points also depends on the number and type of users to be serviced. There are two main advantages to using a wireless LAN: it costs less to install and maintain, and large areas can be covered by adding wireless LAN access points to boost or extend the range. That said, in large facilities more network access points or wireless network access points may be necessary. Wireless LAN access points are positioned according to site inspection recommendations. On the whole, network access points wirelessly connect users to a corporate network while access points deliver transparent bridging and roaming capabilities. Access point function provides network infrastructure connections; these access points are actually the "hub" of a wireless network.

In 802.11 standards, the basic protocol for medium access is referred to as Distributed Coordination Function (DCF). This scheme is based on Carrier Sense Multiple Access in conjunction with Collision Avoidance (CSMA/CA) mechanisms. Retransmission of collided packets is carried out using binary exponential back off rules. The basic 802.11 MAC protocol is the Distributed Coordination Function (DCF) that works according to a listen-before-talk scheme, based on the Carrier Sense Multiple Access (CSMA). Stations deliver MAC Service Data Units (MSDUs) of arbitrary lengths (up to 2304 bytes), after detecting that there is no other transmission in progress on the wireless medium. However, if two stations identify the channel as being free at the same time, a collision occurs.

The 802.11 proposes a Collision Avoidance (CA) mechanism to reduce the probability of such collisions. This is how CA works: before starting a transmission a station performs a back off procedure. It has to keep sensing the channel for an additional random time after detecting the channel as being idle for a minimum duration, called DCF Interface Space (DIFS). The station is allowed to initiate the transmission only if the channel remains idle for this additional random time period. During this transmission time, other stations will have "frozen" their back off counter. The duration of this random time is determined as a multiple of a slot time. Each station maintains a so-called Contention Window $(\mathrm{CW})$, which is used to determine the number of slot times for which a station has to wait before transmission.

For each successful reception of a frame, the receiving station immediately acknowledges the frame reception by sending an acknowledgement frame (ACK). The $\mathrm{CW}$ size increases when a transmission fails-i.e., the transmitted data frame has not been acknowledged. After any unsuccessful transmission attempt, another back off is performed while utilizing the $\mathrm{CW}$ at double capacity. This reduces the collision probability in the event that there are multiple stations attempting to access the channel. The stations that deferred from channel access during the channel's busy period do not select a new random back off time, but rather continue to count down the time of the deferred back off (which is in progress) after interpreting a channel as being idle again. In this manner, stations that defer from channel access because their random back off time was larger than the back off time of other stations are given a higher priority when they 
resume the transmission attempt. After each successful transmission, another random back off is performed by the transmission-completing station.

Channel time is generally wasted owing to errors induced in packets by the channel. In traditional media access protocol, these erroneous packets are assumed to occur because of collision, and more time is wasted in back off slots. It is therefore important to know at the MAC layer the reason for packet loss (that is, whether it is caused by collision or channel error). For the direct measurement at the MAC layer we will be doing an online estimation of collision and packet error probabilities integrated with media access control operations. We will also evaluate performance in more traffic scenarios, such as the hidden node.

In a dense wireless local area network, interference is a significant problem. One of the features of interference losses is that the loss rate experienced will vary greatly between stations in wireless local area networks, depending on their location. This leads to a wellknown predicament called the hidden node problem. Since our estimator makes use of locally measured information, this means each station is able to measure its own local channel quality rather than using some wireless local area network wide measure of interference. The access point (AP) is affected by the interference via the hidden terminal, whereas the wireless local area network client stations are outside the interference range of the hidden node, which leads, ultimately, to disproportionate channel quality within the wireless local area network.

For the direct measurement at the MAC layer we will be doing online estimation of collision and packet error probabilities integrated within media access control operations. At the same time we will evaluate the performance in various traffic scenarios (for instance, the hidden node). The original 802.11 MAC layer uses DCF. However, it has several limitations: (1) if many stations communicate at the same time, many collisions will occur, which will lower the available bandwidth; (2) if we suppose one station wins the access medium, it keeps the medium as long as it wants, which is not desirable, particularly when assuming that, if the accessed station has a low bit rate, it will take longer to send a packet. Because of this delay, other stations may suffer. This translates to a low quality of service (QoS). Thus, we will be incorporating the above online estimation in EDCA.

Other advanced studies in wireless networks are covered in [15], where adaptive rate selection is carried out using a channel sensing mechanism. Analytical modeling for the performance of wireless networks is implemented in [16] for non-saturated channel conditions where the traffic is not backlogged. The other studies on WiMaX and LTE wireless networks can be found in the literature where extensive analysis has been done on both QoS and energy consumption - which are highly essential metrics [17, 18].

In all of these studies, it is evident that for high throughput conditions the measurement of channel quality is of high importance. Better channel conditions are essential for high throughput; however, unlike the wired networks, the channel conditions vary dynamically due to fading. Moreover, the bandwidth is limited. To provide QoS under such limitations is a challenging feat indeed [19]-[20]. The channel measurement mechanism must be optimally efficient so that it will not consume more resources within the wireless network. The developed schemes should be simultaneously resilient and accurate enough to make media access control (MAC) decisions for high throughput, and equilibrium among the users competing for the limited channel resources.

\section{Channel Quality Measurement}

802.11 MAC makes the estimation of channel quality challenging, as packet losses due to colliding transmissions are a feature of normal operations. It is important to understand that the level of collision-induced packet loss is strongly loading dependent. As the number of stations increases, the saturation throughput decreases; this is mainly because 
the collision probability increases as the number of stations increase [11]. However, the channel time is wasted with the number of increased stations [12]. As the number of stations increases the packet loss augments, and the channel time that is wasted multiplies.

We do not yet understand why these packet losses occur, or whether they are caused by collision or channel noise. Therefore, we have to separate the losses owing to collisions from losses owing to channel noise and other sources of error. We would like to achieve this while avoiding the need for specific knowledge of network load, and without making presumptions that all stations are saturated. We can see that station $\mathrm{B}$ is in the range of station $\mathrm{A}$ (and also station $\mathrm{C}$ ), but that station $\mathrm{A}$ and station $\mathrm{C}$ are not in range. For station $\mathrm{A}$, station $\mathrm{C}$ is a hidden node and vice versa. Because both $\mathrm{A}$ and $\mathrm{C}$ are hidden from each other, a collision will occur if both of these stations start to transmit to station B.

By considering the above scenarios, we need to separate the losses due to collisions and losses due to channel noise (and other sources of error). For example, if the channel error is high, it can either shift to a lower rate or select a different channel altogether. On the contrary, if the instantaneous collision probability is high, it can temporarily reduce its number of transmissions until a later time when the load on the channel is low. We would like to achieve the latter while avoiding the need for explicit knowledge of the network load, and without making strong assumptions (e.g., that all stations are saturated). In wireless local area networks, errors are induced in packets both due to collisions and unreliable wireless channels. Estimating quantities such as the probability of collision and probability of channel-induced error will enable the MAC layer to make appropriate decisions.

The SNR (Signal to Noise Ratio) and RSSI (Received Signal Strength Indication) in 802.11 measurements on the physical layer approach are weakly associated with the actual channel behavior at the MAC layer [13]. This means that a direct measurement of channel quality at the MAC layer is obligatory. There is, however, another proposed solution to the problem of separating loss due to collision and loss due to channel noise: in [14] the author modifies 802.11 MAC to send NAK (Negative Acknowledgement) packets when a receiving station infers a channel error. To distinguish the two types of losses, the authors propose the addition of a NAK control frame to DCF (Distributed Coordination Function).

This loss distinguishing method is based on the following observations. The MAC data frame can be partitioned into two functional parts: the header and the payload. The header contains information concerning the frame type, source address, and destination address. If all stations in a WLAN BSS (Basic Service Set) are close enough and can detect one another, a collision occurs only when more than one station sends data to the same time slot. In this case, both the header and body will be corrupted.

If only one station sends a data frame and the frame is lost due to link errors, there is a reasonable chance that the receiver will acquire the header correctly. This is because, in general, the header is much shorter than the entire frame. By observing the content of the header, we can obtain information regarding who has sent the frame, and who is the intended receiver. When a link error occurs, a proper feedback message from the receiver will enable the sender to properly interpret what has happened. We use the NAK frame to notify the sender that the data frame transmission has failed due to a link error.

In essence, if a receiver gathers the correct MAC header, an ACK is sent back. If the MAC body is wrong, a NAK is sent back. If the wrong MAC header is received, the receiver sends nothing. If $A C K$ is received by the sender, the transmission is successful. If a NAK is received, a link error is assumed. If nothing is received, a collision is assumed. The NAK frame can be implemented with exactly the same structure as the ACK frame, except for a one-bit difference in the frame type field in the header. NAKs are sent at the same data rate as those of ACK transmissions. When there is a link error, the transmission of a NAK does not consume more bandwidth; nor does it collide with other frames at all because it occupies the time slot that would have been used by an ACK transmission. 
As mentioned above, the author modifies the 802.11 MAC to send NAK packets when the receiving station infers a channel error. But this is not a rigid MAC layer rule, which is why we are using a standard MAC layer rule to identify the probabilities of packet loss due to collisions, and loss due to channel noise.

\section{Proposed Method}

The procedure for estimation can be performed by first observing the CSMA/CA process; this procedure creates well-defined times in which packet transmissions by a station are admissible, and other times when the packet transmissions are disallowed. This means that transmission can only begin when the medium is idle. To specify the satisfactory transmission times we need to define the following four events. If a station (for example, station-A) has read the medium as idle for DIFS amount of time, it starts counting the slots called as "idle slots".

If station-A has read the medium as being busy due to other station transmitting, it suspends its back off process. In other words, it counts such slots as "other transmissions". Each time station-A transmits on the channel, and receives an acknowledgement it counts them as "successful transmissions". When station-A does not receive an acknowledgement for its transmitted frame, it counts the slots as "unsuccessful transmissions".

By taking the above discussion into consideration, we can calculate the proposed procedure of estimation. Suppose a station observes activity on a channel for a particular time (from 0-M) before updating its estimated parameters. If it transmits $\mathrm{T}$ times out of these, A are successes due to ACK being received. Suppose there are also R slots in which a station does not transmit, and that I of these are idle. First let's make some notations as $\mathrm{pc}=\mathrm{P}$ [collision] and $\mathrm{pe}=\mathrm{P}$ [channel error]. Now we will assume that the probability of at least one other station transmitting in an arbitrary slot does not depend on whether station-A transmits anything.

What this all means: there is a probability that the channel is busy at a slot boundary owing to a transmission by one or more other stations. This assumption is reasonable in a distributed random access MAC scheme (such as CSMA/CA), and is central for wellestablished models of 802.11 operations [5]. One more assumption we are making is that the collision probability of station-A is independent of the back off stage of station-A. According to the above two assumptions, if station-A is not transmitting, then the station reads the medium as busy. This creates the probability that $\mathrm{P}[$ success] is the applicable ratio of successful transmissions to the attempted transmissions.

$$
P[\text { Sucess }]=\left(1-p_{c}\right)\left(1-p_{e}\right)
$$

The collision probability $p_{c}$ is estimated as the proportion of busy slots due to

$$
P_{c}=\frac{R-I}{R}=\frac{\text { other } \text { transmits }}{\text { Idle }+ \text { other transmits }}
$$

Finally we get the probability of channel error as

$$
P_{c}=1-\frac{1-(T-A) / T}{1-P_{c}}
$$

\section{Experimental Results}

To evaluate the accuracy of the above estimated equations, we performed tests over a wide range of network conditions. Standard $802.11 \mathrm{~b}$ parameters were used, which are illustrated in the table below. 
The stations attempt to transmit 1500 byte packets. We will be using a wide spectrum of packet error rates ranging from $1 \%$ to $64 \%$ in our experiment; in addition, varied numbers of stations are used, and the experiment is run for 50 seconds. Figure 1 shows the actual output and estimator output as the number of stations (nodes) is diversified.

Table 1. Standard 802.11b Parameters

\begin{tabular}{|l|l|l|}
\hline Notations & Description & Values \\
\hline MAC & MAC Overhead & 224 Bits \\
\hline HCF & $\begin{array}{l}\text { MAC Header } \\
\text { Checksum Field Size }\end{array}$ & 2 Bytes \\
\hline DIFS & The Time of DIFS & $50 \mu \mathrm{sec}$ \\
\hline SIFS & The time of SIFS & $10 \mu \mathrm{sec}$ \\
\hline ACK_NAK & ACK or NAK frame & $112 \mathrm{bits}$ \\
\hline RTS & RTS frame size & $160 \mathrm{bits}$ \\
\hline CTS & CTS frame size & $112 \mathrm{bits}$ \\
\hline PHY & $\begin{array}{l}\text { Overhead at Physical } \\
\text { Layer }\end{array}$ & $192 \mu \mathrm{sec}$ \\
\hline
\end{tabular}

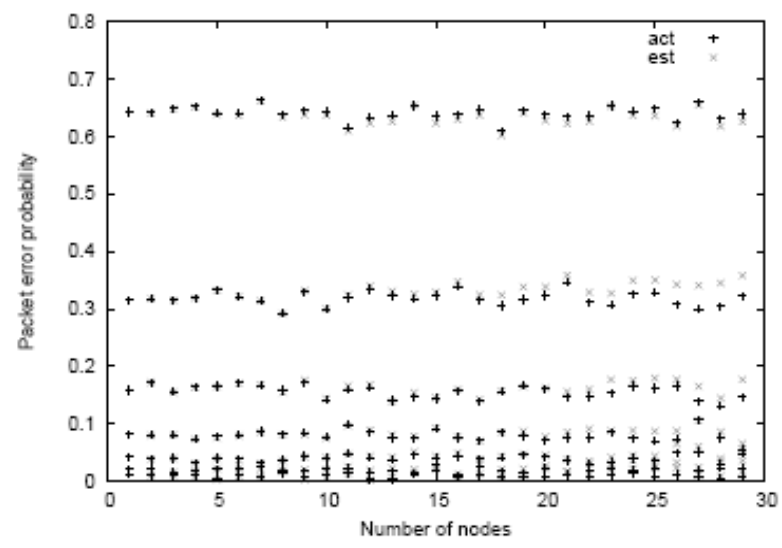

Figure 1. Performance of Estimator for Various Packet Error Probabilities

Figure 1 shows the performance of our estimator with respect to actual packet error probabilities for various stations. From the above figure we can deduce that the estimated output is not exactly the same as the actual output; this is mainly because of the finite nature of the experiment (50 seconds in duration). Another measurement of estimator accuracy occurs by assessing the actual error probability against the estimated (from the above equations that we generated theoretically) packet error probability. This result is shown in Figure 2.

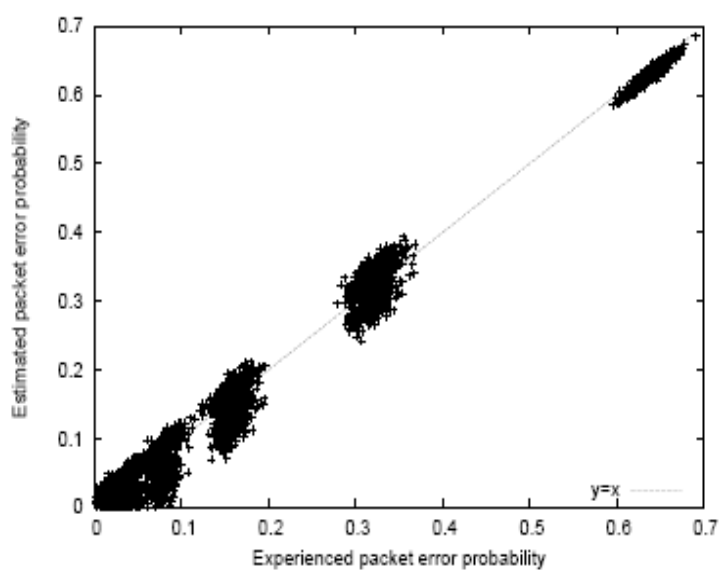

Figure 2. Accuracy of the Estimator 
Figure 2 shows the accuracy of the estimator when we plot the actual error probability against the estimated packet error probability. From this result we can detect some spread when the error probability between estimated and actual is negligible. This means that the difference between the actual packet error probability and estimated error packet probability is quite small. We can see the spread in most parts of our experiment, which ranges from $1 \%$ to $64 \%$. Ultimately, our estimator is remarkably accurate within a diverse range of conditions. The estimator performs similarly under varying offered loads.

We can observe that our estimator converges to the error probability faster than the error probability converges to its limit. The graph illustrates the limit of convergence as 0.08 .

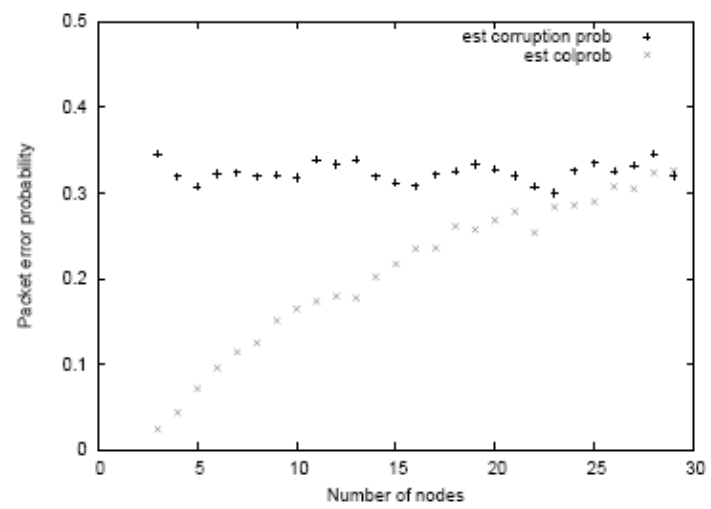

Figure 3. Estimated Corruption Probability as the Number of Normal Nodes Varies

Figure 3 shows that as the number of nodes increase, the collision probability is amplified within the range of $0 \%-30 \%$. The error probability, which is determined by the hidden node transmissions, remains constant because there is only one hidden node in our exemplum scenario. Yet if we suppose that the nodes are constant, then the collision probability will be constant.

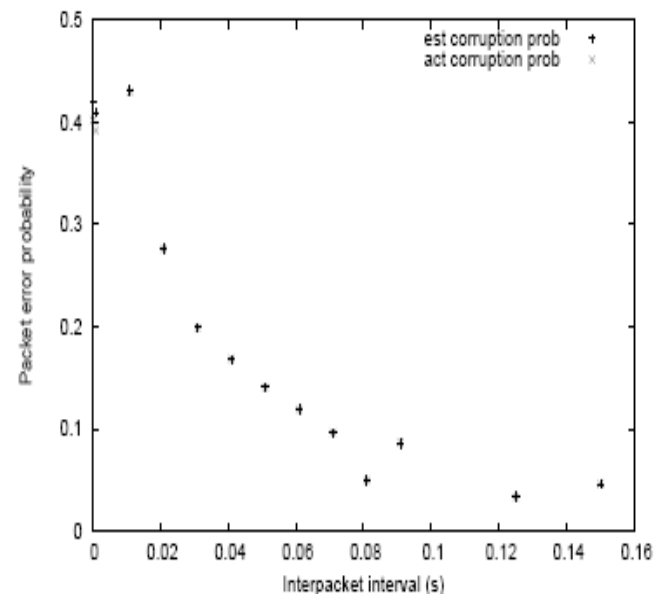

Figure 4. Estimated vs. Actual Corruption Probabilities for One Node as Load on Hidden-Node varies

Figure 4 shows the estimated versus actual collision probabilities for one node as the load on the hidden node is varied. By varying the offered load at the hidden node while keeping the number of stations (nodes) in the wireless local area network constant, we can note that the packet error rate rapidly decreases (see graph below) as the packet's arrival time in the hidden node increases. In essence, if we increase the time interval between the 
packets on hidden nodes, then the packet error will automatically decrease as the time increases.

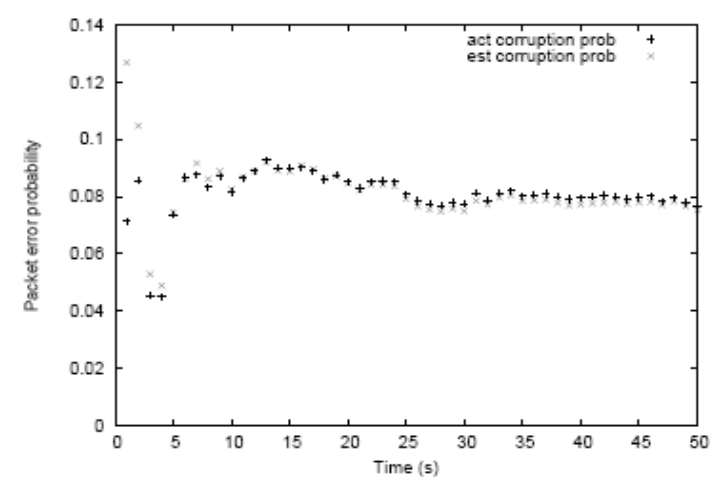

Figure 5. Convergence of Estimator

From Figure 5, we can confirm that our estimator converges to the error probability faster than the error probability converges to its limit, as mentioned before that the limit of convergence is 0.08 . We observed that, for a moderately loaded $802.11 \mathrm{~b}$ network, twenty seconds is enough time for the estimator to converge. By observing the above graphs, we can verify that our estimated equations for packet error probability are accurate when compared with the actual packet error probability.

\section{Conclusion}

In this paper we presented a novel technique for accurately estimating the proportions of packet loss due to collisions, and from other sources of loss due to channel noise and hidden nodes. We arrived at this conclusion while avoiding the need for specific knowledge of the network load, and without making strong assumptions that all stations are saturated. The required measurements are available in standard hardware, as they are required for CSMA/CA operations. The original 802.11 MAC layer uses DCF. It has several limitations (as outlined in section I), which can translate to a low quality of service (QoS). Therefore, we will be incorporating the above online estimation in EDCA (Enhanced Distributed Channel Access), otherwise known as 802.11e. This IEEE802.11e is an approved standard that delineates quality of service enhancements for LAN applications.

\section{Acknowledgement}

The authors would like to extend their sincere thanks to Prince Sultan University (PSU), Riyadh, K.S.A. for its support to the project.

\section{References}

[1] S. Mundra, V. J. Ribeiro, "Throughput improvement of IEEE 802.11 using adaptive slot size", Proc. Communication Systems and Networks, (2014) January, pp. 1-8, Bangalore.

[2] A. B. Makhlouf and M. Hamdi, "Dynamic multi-user access scheme for IEEE 802.11 WLAN channels", Proc. Wireless Communications and Mobile Computing, (2014) August, pp. 18-23, Cyprus.

[3] K. A. Meerja, A. Shami, "Analysis of enhanced collision avoidance scheme proposed for IEEE 802.11eenhanced distributed channel access protocol”, IEEE Transactions on Mobile Computing, vol. 8, no. 10, (2009) October, pp. 1353-1367,.

[4] D. J. Dechene, K. A. Meerja and A. Shami, "Performance evaluation of MIMO-aware media access control protocol", Physical Communication, (2009) September, vol. 2, no. 3, pp. 204-216.

[5] K. A. Meerja and A. Shami, "Enhancing channel utilization by improving media access coordination in wireless local area networks", Wireless Communications and Mobile Computing Journal, vol. 8, no. 2, pp. 155-169, (2008) February. 
[6] K. A. Meerja and A. Shami, "Analysis of new distributed media access control schemes for IEEE 802.11 wireless local area networks", IEEE Transactions on Vehicular Technology, vol. 56, no. 4, pp. 17971812, (2007) July.

[7] D. J. Leith and P. Clifford, "A Self-Managed Distributed Channel Selection Algorithm for WLANs", Proc. IEEE RAWNET, (2006) Boston.

[8] A. Mishra, V. Brik, S. Banerjee, A. Srinivasan and W. Arbaugh, "A Client driven Approach for Channel Management in Wireless LANs", Proc. IEEE INFOCOM, (2006), Barcelona.

[9] B. Kauffmann, F. Baccelli, A. Chaintreau, K. Papagiannaki and C. Diot, "Self Organization of Interfering 802.11 Wireless Access Networks", INRIA Technical Report, (2005) August.

[10] D. Aguayo, J. Bicket, S. Biswa, G. Judd and R. Morris, "Link-level measurements from an 802.11b mesh network", Proc. ACM SIGCOMM, (2004), Boston.

[11] G. Bianchi, "Performance analysis of IEEE 802.11 distributed coordination function", IEEE Journal on Selected Areas in Communications, vol. 18, no. 3, (2000) March, pp. 535-547.

[12] M. Heusse, F. Rousseau, R. Guillier and A. Duda, "Idle sense: an optimal access method for high throughput and fairness in rate diverse wireless LANs", Proc. IEEE SIGCOMM, (2005), Philadelphia.

[13] D. Qiao and Sunghyun Choi, "Good put Enhancement of IEEE 802.11a Wireless LAN via Link Adaptation", Proc. IEEE ICC, (2001), Finland.

[14] Q. Pang, S. C. Liew, and V. C. M. Leung, "Design of an Effective Loss- Distinguishable MAC Protocol for 802.11 WLAN", IEEE Communications Letters, vol. 9, no. 9, (2005) September, pp. 781- 783.

[15] G. Yang, H. Zheng, J. Zhao and V. O. K. Li, "Adaptive Channel Selection through Collaborative Sensing", Proc. IEEE ICC, (2006), Turkey.

[16] D. Malone, K. Duffy and D. J. Leith, "Modeling the 802.11 distributed coordination function in nonsaturated heterogeneous condition”, IEEE/ACM Transactions on Networking, (2007).

[17] X. Bai, A. Shami, and Y. Ye, "Robust QoS Control for Single Carrier PMP Mode IEEE 802.16 System," IEEE Transactions on Mobile Computing, vol. 7, no. 4, (2008) April, pp. 416-429.

[18] D. Dechene and A. Shami, "Energy-Aware Resource Allocation Strategies for LTE Uplink with Synchronous HARQ Constraints," IEEE Transactions on Mobile Computing, vol. 13, no. 2, (2014) February, pp. 422-433.

[19] M. Mirahmadi and A. Shami, "Traffic-prediction-assisted dynamic bandwidth assignment for hybrid optical wireless networks," Computer Networks, vol. 56, no. 1, (2012), pp. 244-259.

[20] D.J. Dechene and A. Shami, "Energy Efficient Resource Allocation in SC-FDMA Uplink with Synchronous HARQ Constraints," IEEE International Conference on Communications (ICC), (2011) June, pp.1-5.

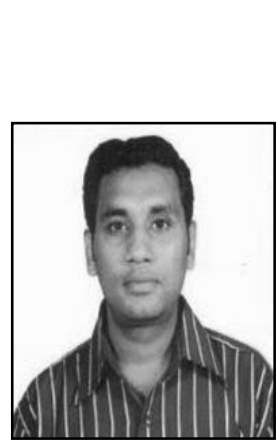

\section{Authors}

Shafi Shahsavar Mirza, was born in Guntur, India, in 1978. He received the B.E degree from University of Karnataka in 2000 and the M.S degree from the University of Western Ontario London, Canada in 2008, both in electronics and communication engineering. He is currently pursuing the Ph.D. degree with the Department of Electronics and Communication Engineering, in KL University, India in collaboration with the University of Western Ontario. London, Ontario, Canada. His research interests include wireless communication, Biomedical Instrumentation and Signal Processing.

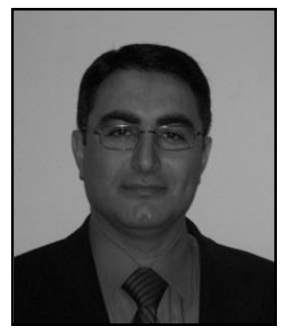

Khaled Almustafa, received his B.E.Sc. in Electrical Engineering, M.E.Sc and Ph.D. in Wireless Communication from the University of Western Ontario, London, Ontario, Canada in 2003, 2004 and 2007 respectively. He is currently the Chairman of the Department of Communication and Networks Engineering, College of Engineering at Prince Sultan University (PSU), Riyadh, K.S.A. His research interests include error performance evaluation of MIMO communication systems in partially known channels, adaptive modulation, and Channel Security, ALPR Image Processing models, Wireless LAN and optical-wireless access network. 
International Journal of Future Generation Communication and Networking Vol. 8, No. 2 (2015) 